\title{
Multiplicity of Positive Periodic Solutions of Singular Semipositone Third-Order Boundary Value Problems
}

\author{
Yigang Sun \\ Department of Applied Mathematics, Hohai University, Nanjing 210098, China \\ Correspondence should be addressed to Yigang Sun, hongyimingsun@163.com
}

Received 2 July 2007; Accepted 13 December 2007

Recommended by Colin Rogers

We establish the existence of multiple positive solutions for a singular nonlinear third-order periodic boundary value problem. We are mainly interested in the semipositone case. The proof relies on a nonlinear alternative principle of Leray-Schauder, together with a truncation technique.

Copyright (C) 2008 Yigang Sun. This is an open access article distributed under the Creative Commons Attribution License, which permits unrestricted use, distribution, and reproduction in any medium, provided the original work is properly cited.

\section{Introduction}

In this paper, we study the existence and multiplicity of positive periodic solutions of the following singular nonlinear third-order periodic boundary value problem:

$$
\begin{array}{cc}
u^{\prime \prime \prime}+\rho^{3} u=f(t, u), & 0 \leq t \leq 2 \pi \\
u^{(i)}(0)=u^{(i)}(2 \pi), & i=0,1,2 .
\end{array}
$$

Here $\rho \in(0,1 / \sqrt{3})$ is a positive constant and $f(t, u)$ is continuous in $(t, u)$ and $2 \pi$ - is periodic in $t$. We are mainly interested in the case that $f(t, u)$ may be singular at $u=0$ and satisfies the following semipositone condition:

$\left(\mathrm{G}_{1}\right)$ There exists a constant $L>0$ such that $F(t, u)=f(t, u)+L \geq 0$ for all $(t, u) \in[0,2 \pi] \times[0, \infty)$.

During the last two decades, singular periodic problems have deserved the attention of many researchers [1-8]. Third-order boundary value problems have also been studied in [9-11]. For the problem (1.1), we recall the following results. In [12], by using Schauder fixedpoint theorem, together with perturbation technique, it was established the existence of at least one positive solution under some suitable conditions of $f(t, u)$. One hard restriction in [12] was the monotonicity on $f(t, u)$. In [13], this restricted condition was removed and the existence 
of multiple positive solutions was obtained by using the fixed-point index theory. Recently, instead of Schauder fixed-point theorem and fixed-point index theory, Chu and Zhou [10] employed a nonlinear alternative principle of Leray-Schauder and a fixed-point theorem in cones due to Krasnoselskii [14] to study problem (1.1). It was proved that (1.1) has at least two positive solutions for the positone case and has at least one positive solution for the semipositone case.

For the convenience of the reader, we recall the following result obtained in [10] for the semipositone case.

Theorem 1.1. Suppose that $\left(G_{1}\right)$ is satisfied. Furthermore, it is assumed that

$\left(\mathrm{G}_{2}\right)$ there exist continuous nonnegative functions $g(u)$ and $h(u)$ on $(0, \infty)$ such that

$$
F(t, u) \leq g(u)+h(u) \quad \forall(t, u) \in[0,2 \pi] \times(0, \infty)
$$

and $g(u)>0$ is nonincreasing and $h(u) / g(u)$ is nondecreasing in $u$;

$\left(G_{3}\right)$ there exist continuous, nonnegative functions $g_{1}(u)$ and $h_{1}(u)$ on $(0, \infty)$ such that

$$
F(t, u) \geq g_{1}(u)+h_{1}(u) \quad \forall(t, u) \in[0,2 \pi] \times(0, \infty)
$$

and $g_{1}(u)>0$ is nonincreasing and $h_{1}(u) / g_{1}(u)$ is nondecreasing in $u$;

$\left(\mathrm{G}_{4}\right)$ there exists $r>\rho \omega / \sigma$ such that

$$
\frac{r}{g(\sigma r / \rho-\omega)\{1+(h(r / \rho-\omega) / g(r / \rho-\omega))\}}>\frac{1}{\rho^{2}}
$$

where $\omega=L / \rho^{3}, \sigma=m / M$ will be given in Section 2.

$\left(\mathrm{G}_{5}\right)$ There exists $R>r$ such that

$$
\frac{R}{g_{1}(R / \rho-\omega)\left\{1+\left(h_{1}(\sigma R / \rho-\omega) / g_{1}(\sigma R / \rho-\omega)\right)\right\}} \leq \frac{1}{\rho^{2}} .
$$

Then problem (1.1) has a positive solution $u$ with $u(t)>0$ for $t \in[0,2 \pi]$ and $r / \rho<\|u+\omega\|<R / \rho$.

The rest of this paper is organized as follows. In Section 2, some preliminary results will be given. In Section 3, we will state and prove the main results. Furthermore, an illustrating example will be given.

\section{Preliminaries}

In this section, we present some preliminary results. First, as in [13], we transform the problem into an integral equation.

For any function $u \in \mathbb{C}[0,2 \pi]$, we define the operator

$$
(J u)(t)=\int_{0}^{2 \pi} g(t, x) u(x) d x
$$

where

$$
g(t, x)= \begin{cases}\frac{e^{-\rho(t-x)}}{1-e^{-2 \pi \rho}}, & 0 \leq x \leq t \leq 2 \pi \\ \frac{e^{-\rho(2 \pi+t-x)}}{1-e^{-2 \pi \rho}}, & 0 \leq t \leq x \leq 2 \pi .\end{cases}
$$


By a direct calculation, we can easily obtain

$$
\int_{0}^{2 \pi} g(t, x) d x=\frac{1}{\rho}
$$

Next we consider the equation

$$
u^{\prime \prime}-\rho u^{\prime}+\rho^{2} u=F(t, J(u(t))-\omega), \quad 0 \leq t \leq 2 \pi
$$

with the following periodic boundary condition:

$$
u(0)=u(2 \pi), \quad u^{\prime}(0)=u^{\prime}(2 \pi)
$$

If $u(t)>L / \rho^{2}$, for all $t \in[0,2 \pi]$, is a solution of problem (2.4)-(2.5), it is easy to verify that $y(t)=J(u(t))-\omega$ is a positive solution of problem (1.1) (for more details, see [10]). Consequently, we will concentrate our study on problem (2.4)-(2.5).

Lemma 2.1 (see [12]). The boundary value problem (2.4)-(2.5) is equivalent to integral equation

$$
u(t)=\int_{0}^{2 \pi} G(t, s) F(s, J(u(s))-\omega) d s,
$$

where

$$
\begin{aligned}
& G(t, s) \\
& \quad= \begin{cases}\frac{2 e^{(\rho / 2)(t-s)}\left[\sin (\sqrt{3} / 2) \rho(2 \pi-t+s)+e^{-\rho \pi} \sin (\sqrt{3} / 2) \rho(t-s)\right]}{\sqrt{3} \rho\left(e^{\rho \pi}+e^{-\rho \pi}-2 \cos \sqrt{3} \rho \pi\right)}, & 0 \leq s \leq t \leq 2 \pi, \\
\frac{2 e^{(\rho / 2)(2 \pi+t-s)}\left[\sin (\sqrt{3} / 2) \rho(s-t)+e^{-\rho \pi} \sin (\sqrt{3} / 2) \rho(2 \pi-s+t)\right]}{\sqrt{3} \rho\left(e^{\rho \pi}+e^{-\rho \pi}-2 \cos \sqrt{3} \rho \pi\right)}, & 0 \leq t \leq s \leq 2 \pi .\end{cases}
\end{aligned}
$$

Moreover, we have the estimates

$$
0<m=\frac{2 \sin (\sqrt{3} \rho \pi)}{\sqrt{3} \rho\left(e^{\rho \pi}+1\right)^{2}} \leq G(t, s) \leq \frac{2}{\sqrt{3} \rho \sin (\sqrt{3} \rho \pi)}=M, \quad \forall s, t \in[0,2 \pi]
$$

In applications below, we take $X=\mathbb{C}[0,2 \pi]$ with the supremum norm $\|\cdot\|$ and we define an operator $T: X \rightarrow X$ by

$$
(T u)(t)=\int_{0}^{2 \pi} G(t, s) F(s,(J u)(s)) d s
$$

where $F:[0,2 \pi] \times \mathbb{R} \rightarrow[0, \infty)$ is a continuous function. It is easy to see that $T$ is continuous and completely continuous. 


\section{Main results}

In this section, we state and prove the main results of this paper.

Theorem 3.1. Suppose that $f(t, u)$ satisfies $\left(G_{1}\right)-\left(G_{5}\right)$. In addition, suppose that

$\left(\mathrm{G}_{6}\right)$ there exists a nonincreasing positive continuous function $g_{0}(u)$ on $(0,+\infty)$ and a constant $R_{0}$ such that $f(t, u) \geq g_{0}(u)$ for $(t, u) \in[0,2 \pi] \times\left(0, R_{0}\right]$, where $g_{0}(u)$ satisfies the strong force condition, that is, $\lim _{u \rightarrow 0^{+}} g_{0}(u)=+\infty$ and $\lim _{x \rightarrow 0^{+}} \int_{x}^{R_{0}} g_{0}(u) d u=+\infty$.

Then problem(1.1) has at least one positive periodic solution $\bar{u}$ with $\omega<\|\bar{u}+\omega\|<r / \rho$.

Proof. We only need to show that problem (2.4)-(2.5) has a solution $u(t)>L / \rho^{2}$ and $L / \rho^{2}<$ $\|u\|<r$, for all $t \in[0,2 \pi]$. To do so, we will use the Leray-Schauder alternative principle, together with a truncation technique.

Let $\mathbb{N}_{0}=\left\{n_{0}, n_{0}+1, \ldots\right\}$, where $n_{0} \in \mathbb{N}$ is chosen such that $1 / n_{0}<\sigma r-\left(L / \rho^{2}\right)$ and

$$
\frac{1}{\rho^{2}} g(\sigma r / \rho-\omega)\left(1+\frac{h(r / \rho-\omega)}{g(r / \rho-\omega)}\right)+\frac{1}{n_{0}}<r
$$

For $\lambda \in(0,1)$, consider the family of equations

$$
u^{\prime \prime}-\rho u^{\prime}+\rho^{2} u=\lambda F_{n}(t, J(u(t))-\omega)+\frac{\rho^{2}}{n}, \quad n \in \mathbb{N}_{0}
$$

where

$$
F_{n}(t, x)= \begin{cases}F(t, x), & x \geq \frac{1}{n \rho} \\ F\left(t, \frac{1}{n \rho}\right), & x \leq \frac{1}{n \rho}\end{cases}
$$

Problem (3.2)-(2.5) is equivalent to the following fixed-point problem in $\mathbb{C}[0,2 \pi]$ :

$$
u(t)=\lambda \int_{0}^{2 \pi} G(t, s) F_{n}(s, J(u(s))-\omega) d s+\frac{1}{n} .
$$

We claim that any fixed point $u$ of (3.4) must satisfy $\|u\| \neq r$ for all $\lambda \in[0,1]$. Otherwise, assume that $u$ is a solution of (3.4) for some $\lambda \in[0,1]$ such that $\|u\|=r$. We have

$$
\begin{aligned}
u(t)-\frac{1}{n} & =\lambda \int_{0}^{2 \pi} G(t, s) F_{n}(s, J(u(s))-\omega) d s \\
& \geq \lambda m \int_{0}^{2 \pi} F_{n}(s, J(u(s))-\omega) d s \\
& =\sigma M \lambda \int_{0}^{2 \pi} F_{n}(s, J(u(s))-\omega) d s \\
& \geq \sigma \max _{t}\left\{\lambda \int_{0}^{2 \pi} G(t, s) F_{n}(s, J(u(s))-\omega) d s\right\} \\
& =\sigma\left\|u-\frac{1}{n}\right\| .
\end{aligned}
$$


By $n, n_{0} \in \mathbb{N}_{0}$, it is evident that $1 / n \leq 1 / n_{0}<r$. Hence, for all $t \in[0,2 \pi]$, we have

$$
\begin{gathered}
u(t) \geq \sigma\left\|x-\frac{1}{n}\right\|+\frac{1}{n} \geq \frac{1}{n}, \\
u(t) \geq \sigma\left\|x-\frac{1}{n}\right\|+\frac{1}{n} \geq \sigma\left(\|x\|-\frac{1}{n}\right)+\frac{1}{n}=\sigma\left(r-\frac{1}{n}\right)+\frac{1}{n} \geq \sigma r ;
\end{gathered}
$$

thus, by conditions $\left(\mathrm{G}_{2}\right)$ and $\left(\mathrm{G}_{4}\right)$, we have

$$
\begin{aligned}
u(t) & =\lambda \int_{0}^{2 \pi} G(t, s) F_{n}(s, J(u(s))-\omega) d s+\frac{1}{n} \\
& \leq \int_{0}^{2 \pi} G(t, s) F(s, J(u(s))-\omega) d s+\frac{1}{n} \\
& \leq \int_{0}^{2 \pi} G(t, s) g(J(u(s))-\omega)\left\{1+\frac{h(J(u(s))-\omega)}{g(J(u(s))-\omega)}\right\} d s+\frac{1}{n} \\
& \leq \frac{1}{\rho^{2}} g(\sigma r / \rho-\omega)\left\{1+\frac{h(r / \rho-\omega)}{g(r / \rho-\omega)}\right\}+\frac{1}{n} .
\end{aligned}
$$

Therefore,

$$
r=\|u\| \leq \frac{1}{\rho^{2}} g(\sigma r / \rho-\omega)\left\{1+\frac{h(r / \rho-\omega)}{g(r / \rho-\omega)}\right\}+\frac{1}{n_{0}}
$$

which is a contradiction to the choice of $n_{0}$ and the claim is proved.

From this claim, the nonlinear alternative of Leray-Schauder guarantees that (3.4) has a fixed point, denoted by $u_{n}$ for $n \in \mathbb{N}_{0}$ with the property $\left\|u_{n}\right\|<r$.

In order to pass the solutions $u_{n}$ of the truncation equation (3.2) (with $\lambda=1$ ) to that of the original problem (1.1), we need the fact $\left\|u_{n}^{\prime}\right\| \leq H$ for some constant $H>0$ for all $n \geq n_{0}$. Integrating (3.2) with $\lambda=1$ from 0 to $2 \pi$, we obtain

$$
\rho^{2} \int_{0}^{2 \pi} u_{n}(t) d t=\int_{0}^{2 \pi}\left[F_{n}\left(t, J\left(u_{n}(t)\right)-\omega\right)+\frac{\rho^{2}}{n}\right] d t .
$$

By the periodic boundary condition, $u_{n}^{\prime}\left(t_{0}\right)=0$ for some $t_{0} \in[0,2 \pi]$. Then

$$
\begin{aligned}
\left\|u_{n}^{\prime}\right\| & =\max _{0 \leq t \leq 2 \pi}\left|u_{n}^{\prime}(t)\right|=\max _{0 \leq t \leq 2 \pi}\left|\int_{t_{0}}^{t} u_{n}^{\prime \prime}(s) d s\right| \\
& =\max _{0 \leq t \leq 2 \pi}\left|\int_{t_{0}}^{t}\left[F_{n}\left(s, J\left(u_{n}(s)\right)-\omega\right)+\frac{\rho^{2}}{n}+\rho u_{n}^{\prime \prime}(s)-\rho^{2} u_{n}(s)\right] d s\right| \\
& \leq \int_{0}^{2 \pi}\left[F_{n}\left(s, J\left(u_{n}(s)\right)-\omega\right)+\frac{\rho^{2}}{n}\right] d s+\rho^{2} \int_{0}^{2 \pi} u_{n}(s) d s+\rho\left|u_{n}(t)-u_{n}\left(t_{0}\right)\right| \\
& =2 \rho^{2} \int_{0}^{2 \pi} u_{n}(s) d s+\rho\left|u_{n}(t)-u_{n}\left(t_{0}\right)\right|<4 \pi \rho^{2} r+2 \rho r=: H .
\end{aligned}
$$


In the next lemma, we will show that there exists a constant $\delta>0$ such that

$$
u_{n}(t)-\frac{L}{\rho^{2}} \geq \delta, \quad \forall t \in[0,2 \pi]
$$

for $n$ large enough.

Since $\left\|u_{n}^{(i)}\right\|, i=0,1$ are bounded, $\left\{u_{n}\right\}_{n \in \mathbb{N}_{0}}$ is bounded and equicontinuous family on $[0,2 \pi]$. Now the Arzela-Ascoli theorem guarantees that $\left\{u_{n}\right\}_{n \in \mathbb{N}_{0}}$ has a subsequence, $\left\{u_{n}\right\}_{n \in \mathbb{N}_{n_{k}}}$, converging uniformly to a function $u \in \mathbb{C}[0,2 \pi]$ (obviously, $\delta \leq u(t) \leq r$ ). Furthermore, $u_{n_{k}}$ satisfies the integral equation

$$
u_{n_{k}}(t)=\int_{0}^{2 \pi} G(t, s) F\left(s, J\left(u_{n_{k}}(s)\right)-\omega\right) d s+\frac{1}{n_{k}} .
$$

Letting $k \rightarrow \infty$, we obtain that

$$
u(t)=\int_{0}^{2 \pi} G(t, s) F(s, J(u(s))-\omega) d s,
$$

where the uniform continuity of $F(t, \cdot)$ on $[0,2 \pi] \times[\delta / \rho, r / \rho]$ is used. Hence, $u(t)$ is a positive periodic solution of (2.4)-(2.5).

Finally, it is not difficult to show that $\|u\|<r$, by noting that if $\|u\|=r$, the argument similar to the proof of the first claim will yield a contradiction.

Lemma 3.2. There exists a constant $\delta>0$ such that any solution $u_{n}(t)$ satisfies (3.11) for $n$ large enough.

Proof. The conclusion is established using the strong force condition of $f(t, u)$. By condition $\left(\mathrm{G}_{3}\right)$, there exists $R_{1} \in\left(0, R_{0}\right)$ and a continuous function $\tilde{g}_{0}(\cdot)$ satisfying the strong force condition such that

$$
F\left(t, J\left(u_{n}(t)\right)-\omega\right)-\left(\rho^{2} J\left(u_{n}(t)\right)-\omega\right) \geq \tilde{g}_{0}\left(J\left(u_{n}(t)\right)-\omega\right)>\max \left\{L, \rho^{2} r+\rho H\right\},
$$

for all $(t, u) \in[0,2 \pi] \times\left(0, R_{1}\right]$.

Choose $n_{1} \in \mathbb{N}_{0}$ such that $1 / n_{1}<R_{1}$ and let $\mathbb{N}_{1}=\left\{n_{1}, n_{1}+1, \ldots\right\}$. For $n \in \mathbb{N}_{1}$, let

$$
(0<) \alpha_{n}=\min _{t}\left[u_{n}(t)-\frac{L}{\rho^{2}}\right] \leq \max _{t}\left[u_{n}(t)-\frac{L}{\rho^{2}}\right]=\beta_{n}
$$

First we claim that $\beta_{n}>R_{1}$ for all $n \in \mathbb{N}_{1}$. Otherwise, it is easy to verify that

$$
F_{n}\left(t, J\left(u_{n}(t)\right)-\omega\right)>\rho^{2} r+\rho H
$$

In fact, if $1 / n \leq u_{n}(t)-L / \rho^{2} \leq R_{1}$, following from (3.14), we have

$$
\begin{aligned}
F_{n}\left(t, J\left(u_{n}(t)\right)-\omega\right) & =F\left(t, J\left(u_{n}(t)\right)-\omega\right) \geq \rho^{2}\left(J\left(u_{n}(t)\right)-\omega\right)+\widetilde{g}_{0}\left(J\left(u_{n}(t)\right)-\omega\right) \\
& \geq \widetilde{g}_{0}\left(J\left(u_{n}(t)\right)-\omega\right)>\rho^{2} r+\rho H
\end{aligned}
$$


and if $u_{n}(t)-L / \rho^{2} \leq 1 / n$, we have

$$
F_{n}\left(t, J\left(u_{n}(t)\right)-\omega\right)=F\left(t, \frac{1}{n \rho}\right) \geq \frac{\rho}{n}+\tilde{g}_{0}\left(\frac{1}{n \rho}\right) \geq \tilde{g}_{0}\left(\frac{1}{n \rho}\right)>\rho^{2} r+\rho H .
$$

By (3.16) and integrating (3.2) (with $\lambda=1$ ) from 0 to $2 \pi$, we obtain that

$$
\begin{aligned}
0 & =\int_{0}^{2 \pi}\left[u_{n}^{\prime \prime}-\rho u_{n}^{\prime}+\rho^{2} u_{n}-F_{n}\left(t, J\left(u_{n}(t)\right)-\omega\right)-\frac{\rho^{2}}{n}\right] d t \\
& \leq \rho^{2} \int_{0}^{2 \pi} u_{n}(t) d t-\int_{0}^{2 \pi} F_{n}\left(t, J\left(u_{n}(t)\right)-\omega\right) d t<0 .
\end{aligned}
$$

This is a contradiction and thus the claim is proved.

Next we claimed that $u_{n}^{\prime \prime}(t)>0$, for all $t \in[0,2 \pi]$. Suppose $\alpha_{n}<R_{1}$, that is,

$$
\alpha_{n}=\min _{t}\left[u_{n}(t)-\frac{L}{\rho^{2}}\right]=u_{n}\left(a_{n}\right)-\frac{L}{\rho^{2}}<R_{1}<\max _{t}\left[u_{n}(t)-\frac{L}{\rho^{2}}\right]=\beta_{n} .
$$

So there exists $c_{n} \in[0,2 \pi]$ (without loss of generality, we assume $a_{n}<c_{n}$ ) such that $u_{n}\left(c_{n}\right)-$ $L / \rho^{2}=R_{1}$ and $u_{n}(t) \leq R_{1}+L / \rho^{2}$ for $t \in\left[a_{n}, c_{n}\right]$. It can be checked that

$$
F_{n}\left(t, J\left(u_{n}(t)\right)-\omega\right)>\rho^{2} r+\rho H .
$$

By (3.2) with $\lambda=1$ and (3.21), we can easily obtain that $u_{n}^{\prime \prime}(t)>0$, as $u_{0}^{\prime}\left(a_{n}\right)=0, u_{n}^{\prime}(t)>0$ for all $t \in\left[a_{n}, c_{n}\right]$, and the function $y_{n}:=u_{n}-L / \rho^{2}$ is strictly increasing on $\left[a_{n}, c_{n}\right]$. We use $\xi_{n}$ to denote the inverse function of $y_{n}$ restricted to $\left[a_{n}, c_{n}\right]$.

In order to obtain (3.14), first we will show that

$$
u_{n}(t)-\frac{L}{\rho^{2}} \geq \frac{1}{n \rho}, \quad \text { for some } n \in \mathbb{N}_{1}
$$

Otherwise, there should exist $b_{n} \in\left(a_{n}, c_{n}\right)$ such that $x_{n}\left(b_{n}\right)-L / \rho^{2}=1 / n$ for some $n \in \mathbb{N}_{1}$ and

$$
u_{n}(t)-\frac{L}{\rho^{2}} \leq \frac{1}{n}, \quad \forall a_{n} \leq t \leq b_{n}, \quad \frac{1}{n} \leq u_{n}(t)-\frac{L}{\rho^{2}} \leq R_{1}, \quad \forall b_{n} \leq t \leq c_{n} .
$$

Multiplying (3.2) (with $\lambda=1$ ) by $u_{n}^{\prime}(t)$ and integrating from $b_{n}$ to $c_{n}$, we obtain

$$
\begin{aligned}
\int_{1 / n}^{R_{1}} F\left(\xi_{n}(y), J y\right) d y & =\int_{b_{n}}^{c_{n}} F\left(t, J\left(u_{n}(t)\right)-\omega\right) u_{n}^{\prime}(t) d t=\int_{b_{n}}^{c_{n}} F_{n}\left(t, J\left(u_{n}(t)\right)-\omega\right) u_{n}^{\prime}(t) d t \\
& =\int_{b_{n}}^{c_{n}} u_{n}^{\prime \prime}(t) u_{n}^{\prime}(t) d t-\int_{b_{n}}^{c_{n}}\left(\rho u_{n}^{\prime}+\rho^{2} u_{n}-\frac{\rho^{2}}{n}\right) u_{n}^{\prime}(t) d t .
\end{aligned}
$$

By the facts $\left\|u_{n}\right\|<r$ and $\left\|u_{n}^{\prime}\right\|<H$, one can easily obtain that the last equation is bounded, that is, there exist a constant $\eta>0$ such that

$$
\int_{1 / n}^{R_{1}} F\left(\xi_{n}(y), J y\right) d y \leq \eta
$$


On the other hand, by $\left(G_{3}\right)$ we can choose $n_{2} \in \mathbb{N}_{1}$ large enough such that

$$
\int_{1 / n}^{R_{1}} F\left(\xi_{n}(y), J y\right) d y \geq \int_{1 / n}^{R_{1}} g_{0}(J y) d y>\eta
$$

for all $n \in \mathbb{N}_{2}=\left\{n_{2}, n_{2}+1, \ldots\right\}$. So (3.22) holds for $n \in \mathbb{N}_{2}$.

As a last step, we will show that (3.14) holds. Multiplying (3.2) by $u_{n}^{\prime}(t)$ and integrating from $a_{n}$ to $c_{n}$, we obtain

$$
\begin{aligned}
\int_{\alpha_{n}}^{R_{1}} F\left(\xi_{n}(y), J y\right) d y & =\int_{a_{n}}^{c_{n}} F\left(t, J\left(u_{n}(t)\right)-\omega\right) u_{n}^{\prime}(t) d t=\int_{a_{n}}^{c_{n}} F_{n}\left(t, J\left(u_{n}(t)\right)-\omega\right) u_{n}^{\prime}(t) d t \\
& =\int_{a_{n}}^{c_{n}} u_{n}^{\prime \prime}(t) u_{n}^{\prime}(t) d t-\int_{a_{n}}^{c_{n}}\left(\rho u_{n}^{\prime}+\rho^{2} u_{n}-\frac{\rho^{2}}{n}\right) u_{n}^{\prime}(t) d t .
\end{aligned}
$$

In the same way as in the proof of (3.24), one may readily prove that the right-hand side of the above equality is bounded. On the other hand, by $\left(\mathrm{G}_{3}\right)$ if $n \in \mathbb{N}_{2}$,

$$
\int_{\alpha_{n}}^{R_{1}} F\left(\xi_{n}(y), J y\right) d y \geq \int_{\alpha_{n}}^{R_{1}} g_{0}(J y) d y+M\left(R_{1}-\alpha_{n}\right) \longrightarrow+\infty, \quad\left(\alpha_{n} \longrightarrow 0^{+}\right) .
$$

Thus, the claim is confirmed.

Combined with Theorems 1.1 and 3.1, we can obtain the following multiplicity result.

Theorem 3.3. Suppose that $\left(G_{1}\right)-\left(G_{6}\right)$ are satisfied. Then problem (1.1) has at least two positive periodic solutions $u, \bar{u}$ with $\omega<\|\bar{u}+\omega\|<r / \rho<\|u+\omega\|<R / \rho$.

Corollary 3.4. Let the nonlinearity in (1.1) be

$$
f(t, u)=b(t) u^{-\alpha}+\mu c(t) u^{\beta}+e(t), \quad 0 \leq t \leq 2 \pi
$$

where $\alpha>0$ and $\beta \geq 0, b(t), c(t), e(t)$ are nonnegative continuous functions and $b(t)>0$, for all $t \in[0,2 \pi], \mu>0$ is a positive parameter. Then

(i) if $\beta<1$, problem (1.1) has at least one positive periodic solution for each $\mu>0$;

(ii) if $\beta \geq 1$, problem (1.1) has at least one positive periodic solution for each $0<\mu<\mu_{*}$, where $\mu_{*}$ is some positive constant;

(iii) if $\beta>1$, problem (1.1) has at least two positive periodic solutions for each $0<\mu<\mu_{*}$, here $\mu_{*}$ is the same as in (ii).

Proof. Let $M=\max _{0 \leq t \leq 2 \pi}|e(t)|$ and

$$
g(u)=b_{0} u^{-\alpha}, \quad h(u)=\mu c_{0} u^{\beta}+M, \quad g_{1}(u)=b_{1} u^{-\alpha}, \quad h_{1}(u)=\mu c_{1} u^{\beta},
$$

where

$$
b_{0}=\max _{t} b(t)>0, \quad c_{0}=\max _{t} c(t)>0, \quad b_{1}=\min _{t} b(t)>0, \quad c_{1}=\min _{t} c(t)>0 .
$$


Then conditions $\left(G_{1}\right)-\left(G_{3}\right)$ and $\left(G_{5}\right)$ are satisfied. The existence condition $\left(G_{4}\right)$ becomes

$$
\mu<\frac{\rho^{2} r(\sigma r / \rho-\omega)^{\alpha}-L(r / \rho-\omega)^{\alpha}-b_{0}}{c_{0}(r / \rho-\omega)^{\alpha+\beta}}
$$

for some $r>L / \rho^{2} \sigma$. Hence, problem (1.1) has at least one positive periodic solution for

$$
0<\mu<\mu_{*}=: \sup _{r>0} \frac{\rho^{2} r(\sigma r / \rho-\omega)^{\alpha}-L(r / \rho-\omega)^{\alpha}-b_{0}}{c_{0}(r / \rho-\omega)^{\alpha+\beta}} .
$$

Note that $\mu_{*}=\infty$ if $\beta<1$ and $\mu_{*}<\infty$ if $\beta \geq 1$. We have the desired results (i) and (ii).

If $\beta>1$, then the existence condition $\left(\mathrm{G}_{6}\right)$ becomes

$$
\mu \geq \frac{\rho^{2} R(R / \rho-\omega)^{\alpha}-b_{1}}{c_{1}(\sigma R / \rho-\omega)^{\alpha+\beta}} .
$$

Since $\beta>1$, the right-hand side goes to 0 as $R \rightarrow \infty$. Hence, for any given $0<\mu<\mu_{*}$, it is always possible to find such $R \gg r$ that (3.34) is satisfied. Thus, (1.1) has an additional periodic solution $u$ such that $\|u\|>r$. This implies that (iii) holds.

\section{References}

[1] F. Merdivenci Atici and G. Sh. Guseinov, "On the existence of positive solutions for nonlinear differential equations with periodic boundary conditions," Journal of Computational and Applied Mathematics, vol. 132, no. 2, pp. 341-356, 2001.

[2] J. Chu and M. Li, "Positive periodic solutions of Hill's equations with singular nonlinear perturbations," to appear in Nonlinear Analysis: Theory, Methods \& Applications.

[3] J. Chu, P. J. Torres, and M. Zhang, "Periodic solutions of second order non-autonomous singular dynamical systems," Journal of Differential Equations, vol. 239, no. 1, pp. 196-212, 2007.

[4] M. A. del Pino and R. F. Manásevich, "Infinitely many T-periodic solutions for a problem arising in nonlinear elasticity," Journal of Differential Equations, vol. 103, no. 2, pp. 260-277, 1993.

[5] D. Jiang, J. Chu, and M. Zhang, "Multiplicity of positive periodic solutions to superlinear repulsive singular equations," Journal of Differential Equations, vol. 211, no. 2, pp. 282-302, 2005.

[6] A. C. Lazer and S. Solimini, "On periodic solutions of nonlinear differential equations with singularities," Proceedings of the American Mathematical Society, vol. 99, no. 1, pp. 109-114, 1987.

[7] K. Lan and J. R. L. Webb, "Positive solutions of semilinear differential equations with singularities," Journal of Differential Equations, vol. 148, no. 2, pp. 407-421, 1998.

[8] J. Wang and D. Jiang, "A singular nonlinear second-order periodic boundary value problem," The Tohoku Mathematical Journal, vol. 50, no. 2, pp. 203-210, 1998.

[9] A. Cabada, "The method of lower and upper solutions or second, third, fourth and higher order boundary value problem," Applied Mathematics and Computation, vol. 40, pp. 135-145, 1990.

[10] J. Chu and Z. Zhou, "Positive solutions for singular non-linear third-order periodic boundary value problems," Nonlinear Analysis, vol. 64, no. 7, pp. 1528-1542, 2006.

[11] D. Jiang and R. P. Agarwal, "A uniqueness and existence theorem for a singular third-order boundary value problem on [0, $\infty), "$ Applied Mathematics Letters, vol. 15, no. 4, pp. 445-451, 2002.

[12] L. Kong, S. Wang, and J. Wang, "Positive solution of a singular nonlinear third-order periodic boundary value problem," Journal of Computational and Applied Mathematics, vol. 132, no. 2, pp. 247-253, 2001.

[13] J. Sun and Y. Liu, "Multiple positive solutions of singular third-order periodic boundary value problem," Acta Mathematica Scientia. Series B, vol. 25, no. 1, pp. 81-88, 2005.

[14] M. A. Krasnosel'ski1, Positive Solutions of Operator Equations, P. Noordhoff, Groningen, The Netherlands, 1964. 